\title{
Out of the box: how bees orient in an ambiguous environment
}

\author{
Laura Dittmar, Wolfgang Stürzl ${ }^{1}$, Simon Jetzschke, Marcel Mertes, Norbert Boeddeker* \\ Department of Neurobiology \&' Center of Excellence 'Cognitive Interaction Technology', Bielefeld University, Bielefeld, Germany
}

\section{A R T I C L E I N F O}

\section{Article history:}

Received 16 May 2013

Initial acceptance 28 June 2013

Final acceptance 11 November 2013

Available online 16 January 2014

MS. number: 13-00423R

\section{Keywords:}

bees

features

geometry

homing

learning

orientation

snapshot matching

space perception

view-based navigation

vision
How do bees employ multiple visual cues for homing? They could either combine the available cues using a view-based computational mechanism or pick one cue. We tested these strategies by training honeybees, Apis mellifera carnica, and bumblebees, Bombus terrestris, to locate food in one of the four corners of a box-shaped flight arena, providing multiple and also ambiguous cues. In tests, bees confused the diagonally opposite corners, which looked the same from the inside of the box owing to its rectangular shape and because these corners carried the same local colour cues. These 'rotational errors' indicate that the bees did not use compass information inferred from the geomagnetic field under our experimental conditions. When we then swapped cues between corners, bees preferred corners that had local cues similar to the trained corner, even when the geometric relations were incorrect. Apparently, they relied on views, a finding that we corroborated by computer simulations in which we assumed that bees try to match a memorized view of the goal location with the current view when they return to the box. However, when extra visual cues outside the box were provided, bees were able to resolve the ambiguity and locate the correct corner. We show that this performance cannot be explained by view matching from inside the box. Indeed, the bees adapted their behaviour and actively acquired information by leaving the arena and flying towards the cues outside the box. From there they re-entered the arena at the correct corner, now ignoring local cues that previously dominated their choices. All individuals of both species came up with this new behavioural strategy for solving the problem provided by the local ambiguity within the box. Thus both species seemed to be solving the ambiguous task by using their route memory, which is always available during their natural foraging behaviour.

(C) 2014 The Association for the Study of Animal Behaviour. Published by Elsevier Ltd. All rights reserved.
Several animal species systematically confound the correct corner and the diametrically opposite one in the well-established 'rectangular arena' paradigm (Cheng, 1986; reviewed by Tommasi, Chiandetti, Pecchia, Sovrano, \& Vallortigara, 2012). Such 'rotational errors' have been interpreted as demonstrating the use of the geometry of space for obtaining directional information. Surprisingly, rotational errors can sometimes be observed even in the presence of additional cues that, at least in principle, would clearly allow the animal to identify the correct corner. These observations lead to the hypothesis of a dedicated 'geometric module', which represents space independently of other features, specifying only the target corner's geometric relation to the shape of the environment (Cheng, 1986).

It has been shown recently that insects (Wystrach \& Beugnon, 2009: ants, Gigantiops destructor; Sovrano, Potrich, \& Vallortigara,

\footnotetext{
* Correspondence: N. Boeddeker, Cognitive Neuroscience \& Center of Excellence 'Cognitive Interaction Technology', Bielefeld University, 33615 Bielefeld, Germany. E-mail address: norbert.boeddeker@uni-bielefeld.de (N. Boeddeker).

${ }^{1}$ Present address: Institute of Robotics and Mechatronics, German Aerospace Center (DLR), 82234 Wessling, Germany.
}

2013; Sovrano, Rigosi, \& Vallortigara, 2012: bumblebees, Bombus terrestris), similar to rats and humans (Cheng, 1986; Cheng \& Newcombe, 2005; Pecchia \& Vallortigara, 2010; Vallortigara, 2009; Wang \& Spelke, 2002), make 'rotational errors' in rectangular arenas. They search not only at the rewarded corner, where for example food or an exit was found during training, but also at the diagonally opposite corner, which, of course, is fully equivalent from a purely geometric point of view. These studies suggest that the animals utilize either local (e.g. the angle of the closest corner; Pearce, Good, Jones, \& McGregor, 2004; Tommasi \& Polli, 2004) or global (e.g. the principal axis; Cheng \& Gallistel, 2005) geometric parameters of the environment. However, it has been shown that such errors can be explained by simple view-based navigation strategies without the need for such explicit geometrical representations because the 'geometry' of the environment (as well as its 'features') is implicitly contained in panoramic views, that is, a retinotopic representation (Cheng, 2008; Cheung, Stürzl, Zeil, \& Cheng, 2008; Sheynikhovich, Chavarriaga, Strösslin, Arleo, \& Gerstner, 2009; Stürzl, Cheung, Zeil, \& Cheng, 2008). A basic concept of view-based models is the matching of panoramic images ('image matching'), that is, the comparison of the currently 
perceived image with a reference image of the goal location. Image matching is very successful in describing insect homing behaviour (e.g. Dittmar, Stürzl, Baird, Boeddeker, \& Egelhaaf, 2010; Wystrach \& Beugnon, 2009; Wystrach, Cheng, Sosa, \& Beugnon, 2011; Zeil, 2012). It is based on the idea that homing insects move in such a way that the current visual input matches the 'snapshot' that was stored on previous visits (e.g. Cartwright \& Collett, 1983). Recent evidence suggests that simple snapshot matching could be important in vertebrates as well (Pecchia \& Vallortigara, 2012).

In this study, we extended the well-known 'rectangular box paradigm' by adding visual cues outside the box. Bees were free to explore the different cues and to choose their navigation strategy. This allowed us to observe and to analyse whether and how bees use such distant cues for homing. Using these cues, bees could in principle resolve the ambiguity between opposite corners of the box.

Initially, we had two hypotheses how bees might solve the task. (1) Bees could determine the correct corner by image matching if

(a)

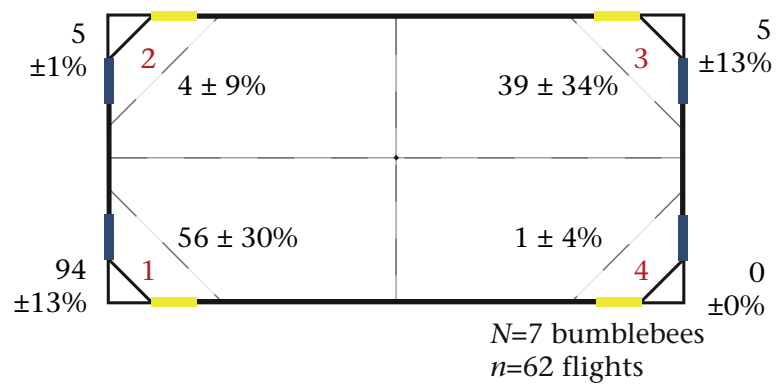

(b)

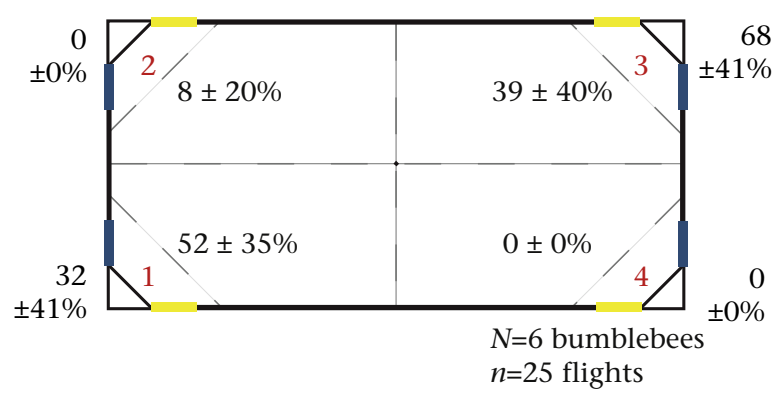

(c)

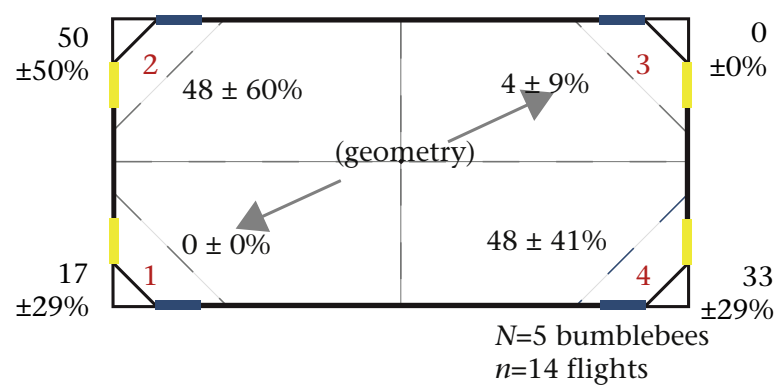

the external cues led to pronounced differences in the images at the opposite corners. To test this hypothesis, we created a computer model of the experimental environment that allowed us to render images from the viewpoint of bees inside the box and to compare the behavioural results with image similarities computed between a panoramic image taken at the rewarded corner in the training configuration and panoramic images in the test situation covering the box in small equidistant steps. (2) Alternatively, bees might be able to detect and recognize the external cues directly, for example by means of local image features (contrast, frequency content, colour, etc.), and use them as a kind of compass providing directional information. The idea behind this is that the task of recognizing a scene would be simpler for the bee when viewing it from the same direction during memory retrieval as during learning. By always adopting a 'standard' orientation with respect to the world at this place, the bee could directly compare retinotopic memories with the current visual input without the need for 'mental rotation'. (d)

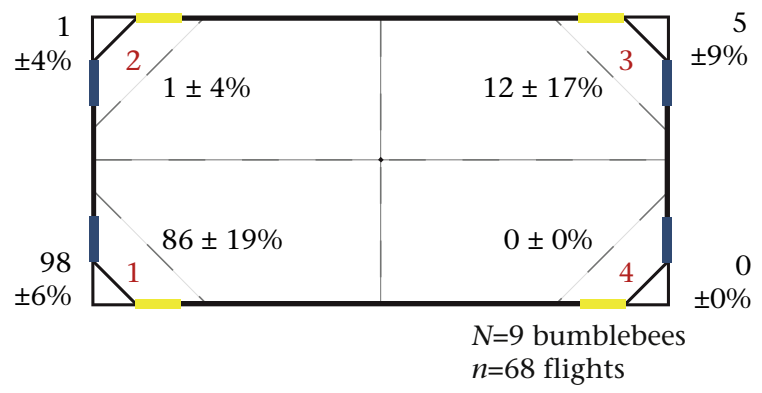

(e)

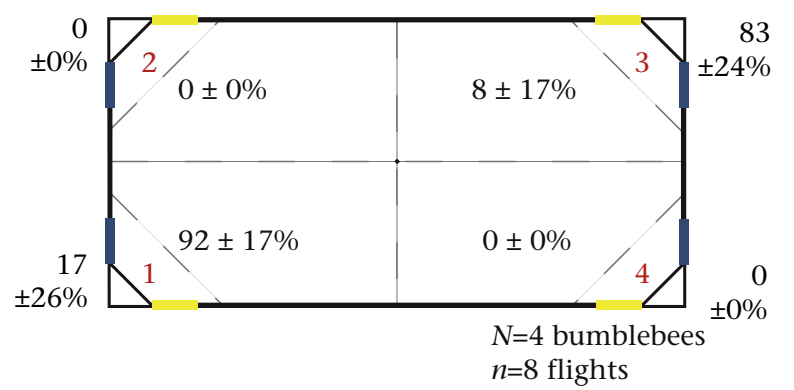

(f)

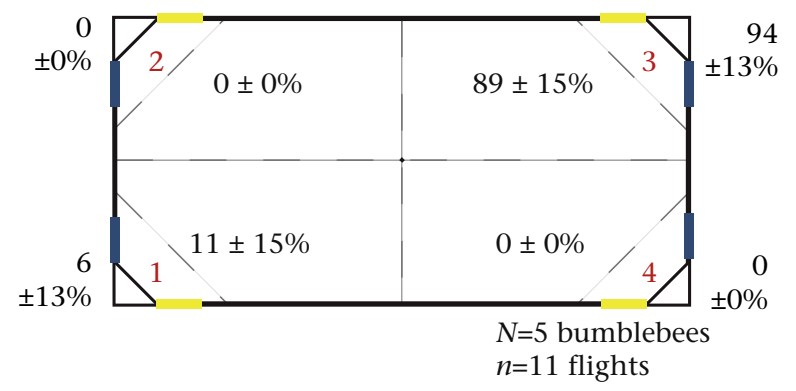

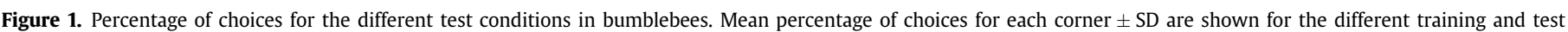

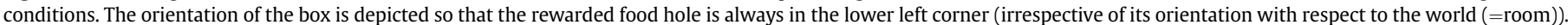

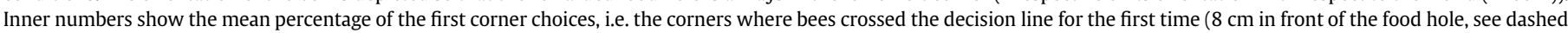

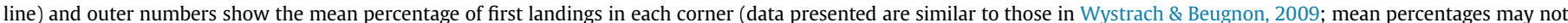

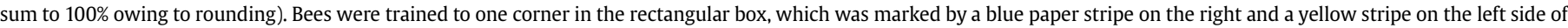

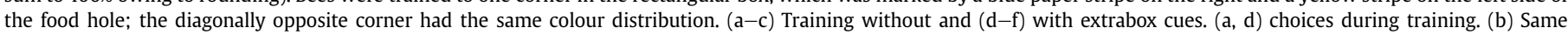

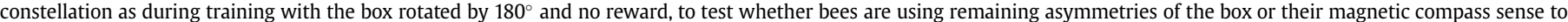

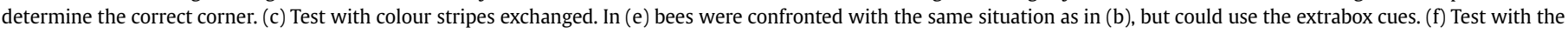
visual cues outside the box (room cues) rotated by $180^{\circ}$ whereas the box was not rotated. 


\section{METHODS}

\section{Experimental Set-up}

Honeybees, Apis mellifera carnica, and medium-sized bumblebees, $B$. terrestris, were trained to visit a food hole containing sugar solution located in one corner of a rectangular box (schematically illustrated in Figs 1,2). The experiments were carried out in an indoor facility at Bielefeld University, Germany, and the research questions, experimental set-up and training procedures were very similar for both species. A beehive located about $130 \mathrm{~m}$ from the experimental room supplied the honeybees used in the experiments. The honeybees entered the room through an open window and were trained to enter a Perspex box where we subsequently trapped them. From here they were transferred in a small tube to the rectangular test box. Bumblebees were obtained from Koppert (Berkel en Rodenrijs, The Netherlands) and housed in custom-built Perspex boxes ( $300 \times 200 \mathrm{~mm}$ and $300 \mathrm{~mm}$ high) that were connected to a flight chamber of the same size, where we provided sugar solution from hanging feeders. We collected bumblebees from this feeding box and transferred them into the test box.

Unlike the honeybees, we were able to train and test the bumblebees in a windowless room where all cues including the

(a)

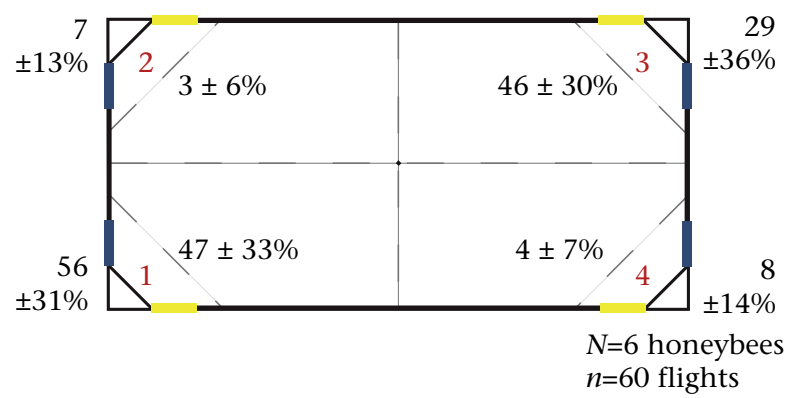

(b)

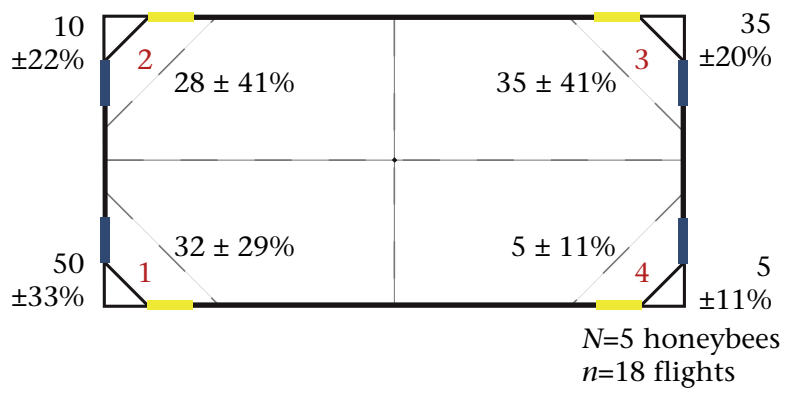

(c)

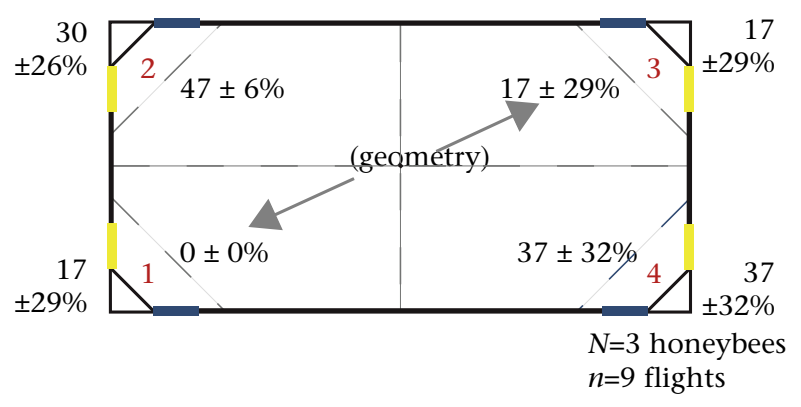

illumination could be fully controlled. This also allowed us to rotate all exterior cues around the rectangular test box (see below). This was a white rectangular box $100 \mathrm{~cm}$ long and $50 \mathrm{~cm}$ wide, with walls $50 \mathrm{~cm}$ (honeybee box) or $30 \mathrm{~cm}$ (bumblebee box) high. The height of the bumblebee box was lower to make the external cues more visible from inside the box. The box was mounted on a swivel that allowed us to spin it around its central vertical axis. In each corner, a little plastic tube served as a food hole (diameter: $75 \mathrm{~mm}$ ). The tube (feeder) was placed on an $11 \mathrm{~cm}$ wide white board, angled $45^{\circ}$ to the walls of the box, at a height of $10 \mathrm{~cm}$ (see e.g. corners in Fig. 1a and Fig. 2a). A green ring ( $3 \mathrm{~cm}$ diameter) marked the position of each of the four food holes, and we also placed horizontal black stripes on the walls (see also Fig. 3a). Two differently coloured paper stripes $(8 \mathrm{~cm}$ wide, $50 \mathrm{~cm}$ (honeybee box) or $30 \mathrm{~cm}$ high (bumblebee box)) were placed $5.5 \mathrm{~cm}$ from the food hole.

During training the short walls of the rectangular box were marked by a yellow stripe and the long walls by a blue stripe (Figs $1 \mathrm{a}, 2 \mathrm{a})$. To distinguish the two diagonally opposite corners (corners numbered 1 and 3 in Figs 1a, 2a) from the neighbouring corners (corners 2 and 4 in Figs 1a, 2a), the bees could also use geometric information (i.e. corners 1 and 3 are characterized by a long wall on the left side and a short wall on the right and for corners 2 and 4 this is the other way round). However, neither geometrical nor

(d)

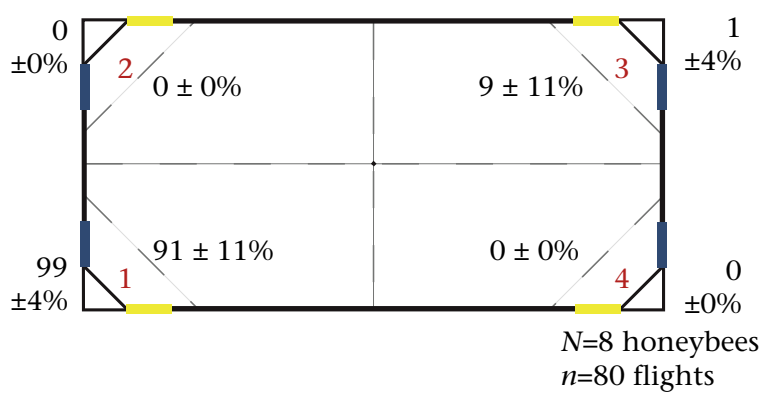

(e)

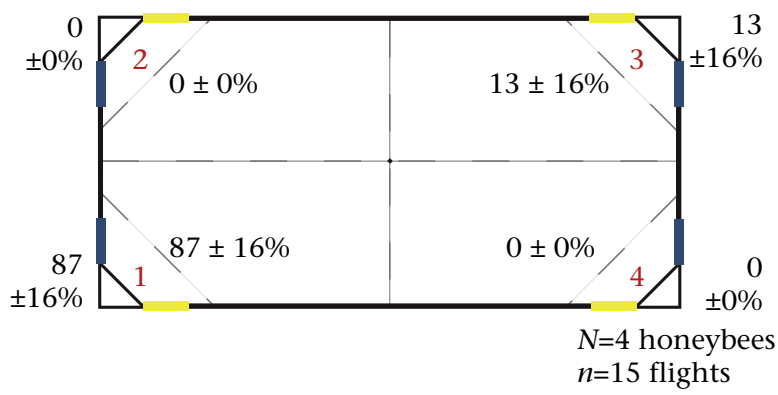

(f)

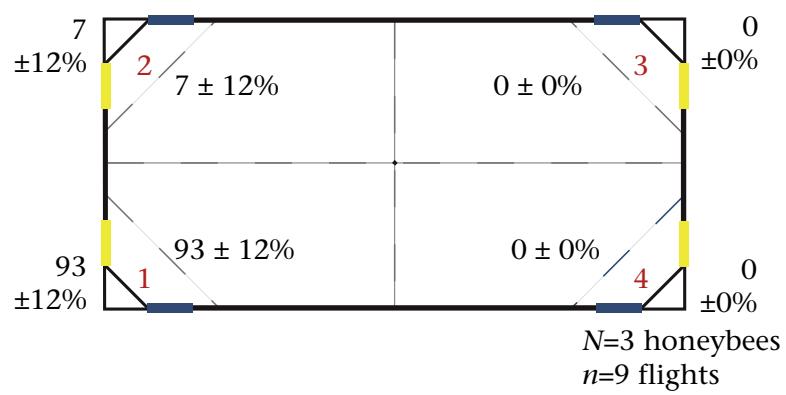

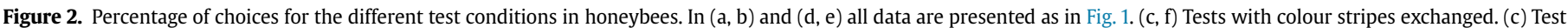

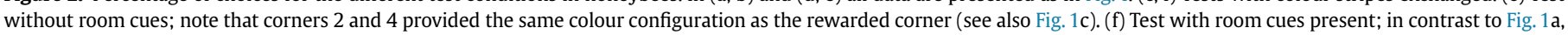
the visual cues outside the box (room cues) were not rotated. 
(a)

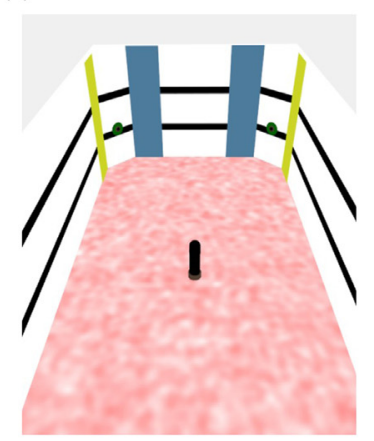

(c)

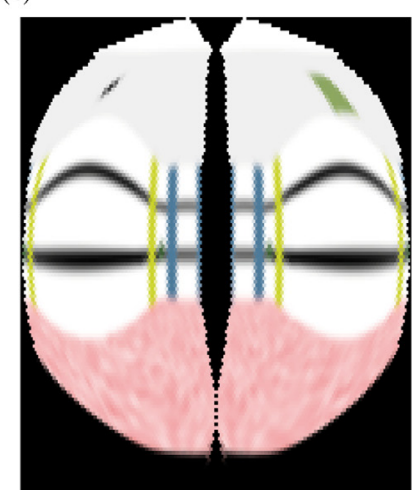

(b)

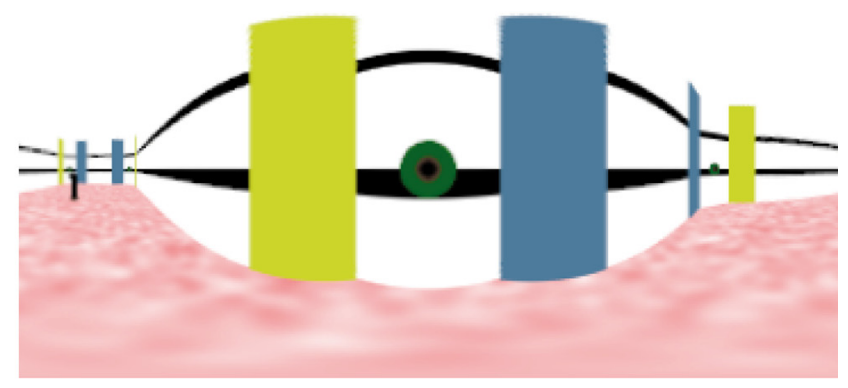

(d)

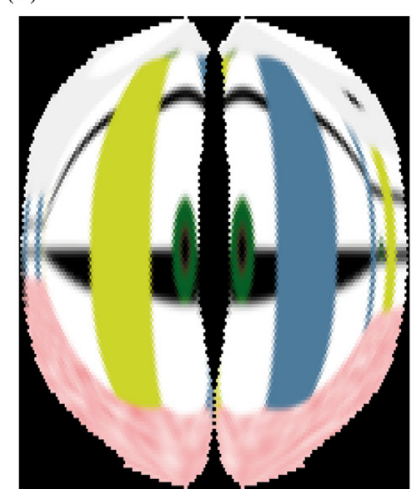

(e)

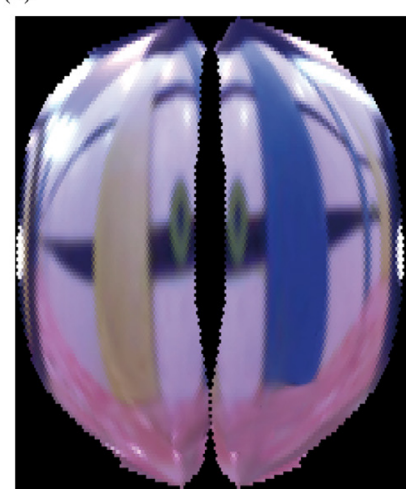

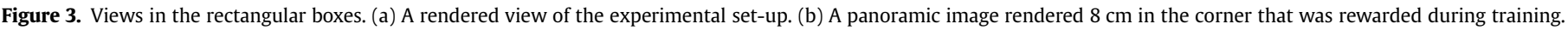

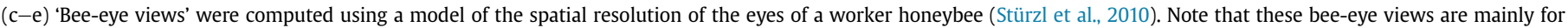

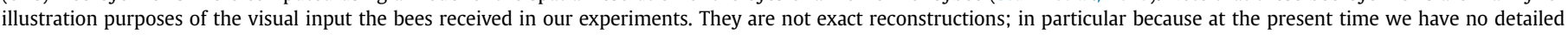

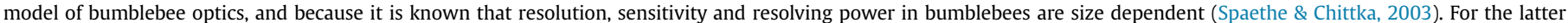

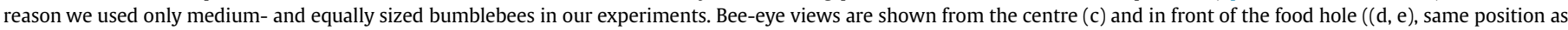

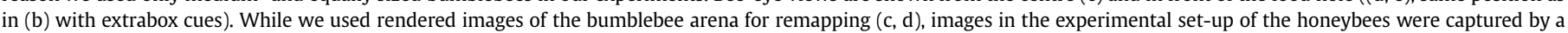

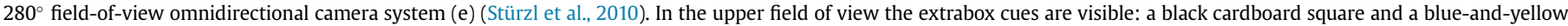
chequerboard (bumblebee arena (c, d)) or a half-white and half-black ceiling (honeybee arena (e)).

colour cues help to distinguish between corners 1 and 3 (or between 2 and 4 ). To facilitate stable flight of bees and to provide additional cues that, at least in principle, would allow the bees to infer the geometry of the box, the floor of the box was covered with a random dot texture and two horizontal black stripes $(1.5 \mathrm{~cm}$ wide, height $10 \mathrm{~cm}$ and $20 \mathrm{~cm}$ ) were attached to the walls. A view of the bumblebee set-up is shown in Fig. 3a.

\section{Experimental Conditions}

The bees were trained and tested in the following two conditions.

\section{Experiment 1, 'local cues only'}

In the first experiment a lid $(100 \mathrm{~cm} \times 50 \mathrm{~cm})$, consisting of a white frame (width $8 \mathrm{~cm}$ ) with white fly mesh (mesh size ca. $1.1 \mathrm{~mm}$ ) was placed on the box. The mesh allowed sufficient light inside the box for the bees to fly and also for the experimenter to see them. At the same time the contrast of objects outside the box was strongly reduced by the lid. We confirmed this by taking panoramic 'bee-eye' views from within the box using a computer model of the eyes of a worker bee (Stürzl, Boeddeker, Dittmar, \& Egelhaaf, 2010). We refer to this condition as the condition providing only 'local' cues to the bees.

\section{Experiment 2, 'additional external cues'}

In the second experiment, bees were trained and tested without the lid. In this case, the bees could use more distant visual cues outside the box to identify the rewarded corner. We refer to these cues as 'extrabox' or 'room' cues.

Honeybees were trained with a box placed in an experimental room providing various cues, the closest ones being a half-white and half-black ceiling $(300 \mathrm{~cm} \times 150 \mathrm{~cm})$ with a dark blue-andorange patterned square $(30 \mathrm{~cm} \times 30 \mathrm{~cm})$ placed in one corner of the ceiling (above the rewarded food hole) to provide a presumably salient cue in the direction of the rewarded corner. A ceiling was placed above the box $100 \mathrm{~cm}$ from the upper edge of the box. The black part of the ceiling provided a large directional cue (placed above the rewarded corner) and covered, when seen from the centre of the box looking upwards, about $55^{\circ}$ in the horizontal and $45^{\circ}$ in the vertical direction.

The bumblebee box was surrounded by a dome of white cloth (height $220 \mathrm{~cm}$, diameter about $195 \mathrm{~cm}$ ) and two salient cues were provided outside the box at the wall of the flight dome. A black cardboard square $(15 \mathrm{~cm} \times 15 \mathrm{~cm})$ was placed at a height of $50 \mathrm{~cm}$ (above the upper edge of the box walls) $65 \mathrm{~cm}$ from the box centre, and a blue-and-yellow chequerboard pattern $(50 \mathrm{~cm} \times 50 \mathrm{~cm})$ was placed to the left of the rewarded corner at a height of $55 \mathrm{~cm}$ and $90 \mathrm{~cm}$ from the box centre. The angle between the vertical at the box centre and both cues was about $60^{\circ}$. From the centre of the box the chequerboard covered about $20^{\circ}$ in elevation and $30^{\circ}$ in azimuth. Indirect illumination was provided by eight Dedo-Lights (DLH4; $150 \mathrm{~W}$ each) placed outside the cloth around the arena and by nine $50 \mathrm{~W}$ halogen lamps from above. All lights ran on DC power and were positioned symmetrically with respect to the arena centre to ensure that the bumblebees could not use 
(a)

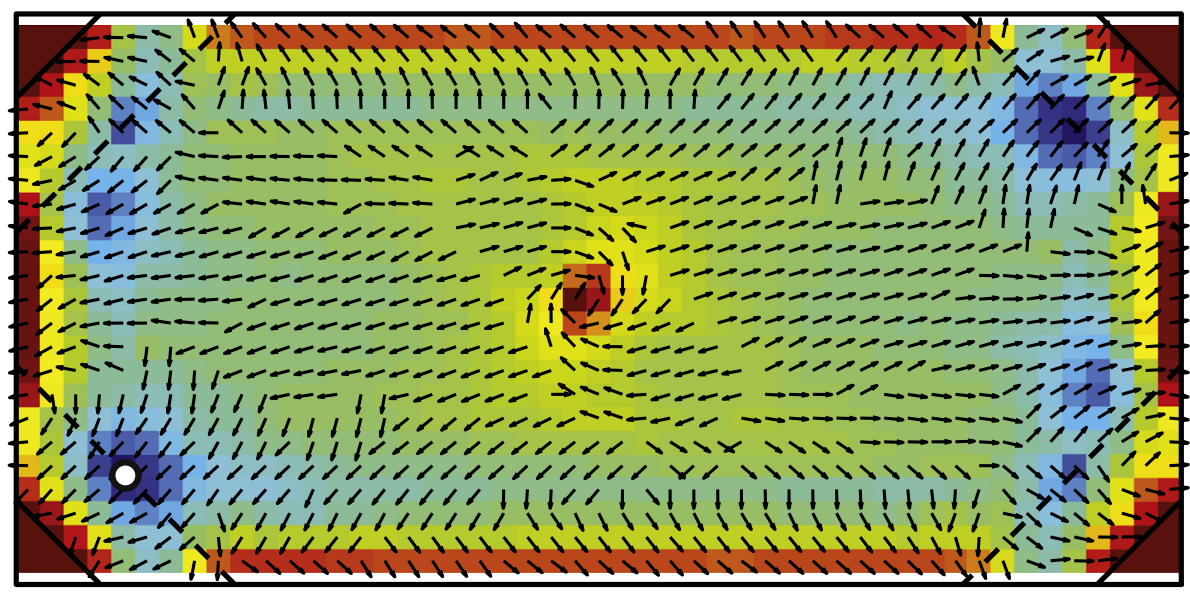

(b)

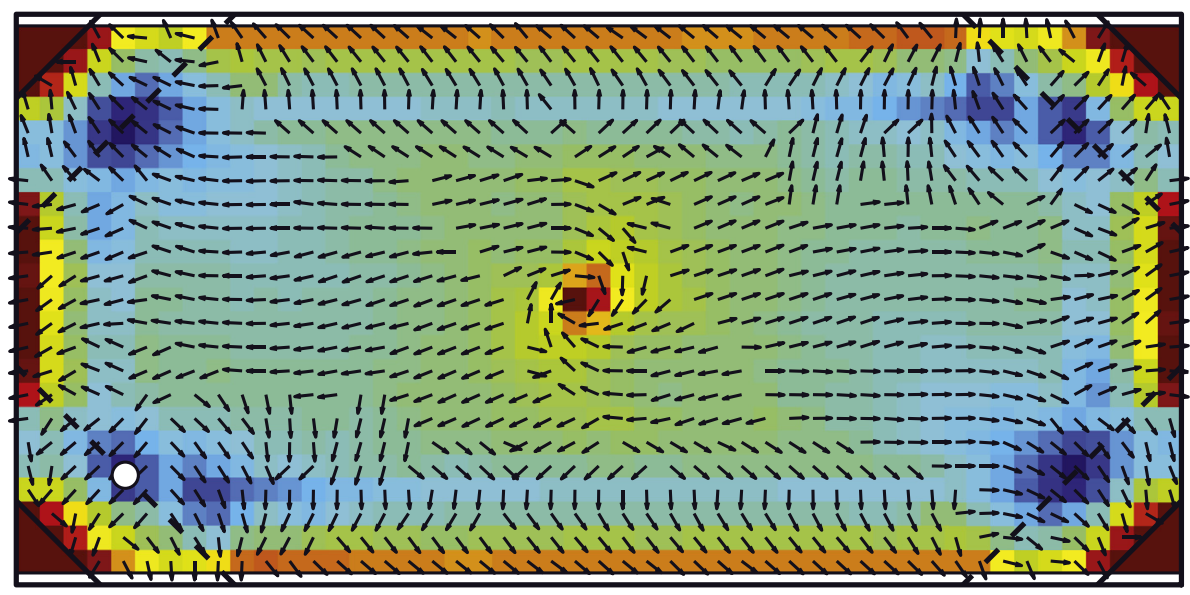

(c)

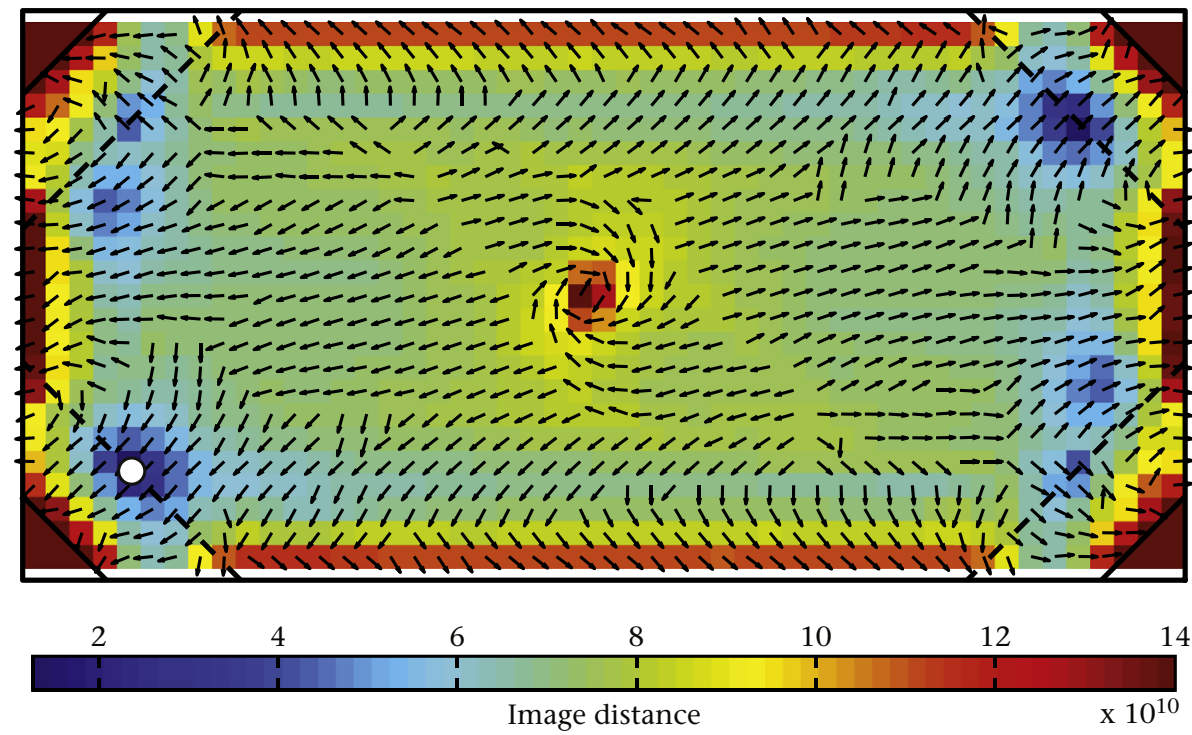

Figure 4. Image similarity maps for the bumblebee box. Similarities between views rendered at the decision line $(8 \mathrm{~cm}$ in front of the food hole) oriented towards the rewarded food hole ('snapshot') and at positions on a two-dimensional grid of $2 \mathrm{~cm}$ spacing $(10 \mathrm{~cm}$ above floor level $)$ were computed using the minimum image distance over all orientations. The white circle indicates the position of the reference snapshot at the goal. The similarity is computed as the sum of the squared pixel differences (for details see Methods). Arrows show the orientation of the best matching view at each position. Image distances are colour-coded and image similarity maps are shown for the bumblebee box for three different experimental conditions: (a) training and test 1 without extrabox cues, (b) test 2 (colour cues exchanged) without extrabox cues, and (c) training and test 1 with extrabox cues. Note that in (c) the effect of extrabox cues on the image similarity is very weak and the pronounced minimum in image distance map in the opposite corner is almost as deep as in the correct one despite the additional features. 
asymmetries in the illumination to distinguish between the corners. During training the orientation of the honeybee and bumblebee boxes (and thus the rewarded corner) was not changed relative to the extrabox cues.

\section{Training and Testing Protocol}

At the beginning of each experiment, individually marked bees were passively transported in a small polystyrene snap cap vial (diameter: $254 \mathrm{~mm}$ ) to the rewarded food hole and allowed to feed. After feeding three to five times, bees were released from the centre of the box at a height of $10 \mathrm{~cm}$ by putting the transport vial in a swivelling holder and opening the lid. We then recorded choices for the four corners. During each trial bees were released in a pseudorandom fashion in one of four starting directions (each pointing towards one corner of the box). During training only one food hole contained a reward, sugar solution; the others were filled with water. After each trial, the whole box and the food hole were cleaned with ethanol (50\%) and the coloured stripes adjacent to the food hole were changed repeatedly to new ones, to avoid any potential olfactory cue. During training with extrabox cues, the bees were allowed to leave and enter the box.

Testing started after the bees completed three successful trials. During the tests all food holes provided water. During testing (every three to four trials), the bees were released in one of two 'new' starting directions randomly facing the middle of one of the short walls. We recorded three parameters (during training and testing): the first choice was defined as the first corner the bees approached (when the bees crossed the 'decision line' at $8 \mathrm{~cm}$ in front of the food hole), the first landing was defined as the first corner the bees touched with their legs, and the flight duration was defined as the time from the start of the flight until the first landing. We performed three different tests. During a control test (test 1), the box was rotated by $180^{\circ}$. In test 2 , we interchanged the coloured stripes of all four corners to put the geometry and the colour cues in conflict (the box was not rotated). In test 3 , the extrabox cues were rotated by $180^{\circ}$ whereas the box was kept in the training orientation. This test was performed only with the bumblebees as we could easily manipulate the available external visual cues in the flight dome around the rectangular box.

\section{Data Analysis}

We calculated the percentage of choices for each corner of all flights of a single bee. When the bees first crossed a very thin line drawn $8 \mathrm{~cm}$ in front of the food hole, this was counted as 'first corner choice'. In addition we also determined the corner of the first landing. For each corner we then calculated the mean percentage and standard deviation of choices and landings across all bees. For each experiment, we compared the distributions of the absolute numbers of first choices for all flights, that is, we analysed whether the choices were equally distributed between the four corners and between the diagonally opposite corners (Pearson chisquare test).

\section{View Reconstruction and Model Simulations}

A computer 3D model of the experimental set-up was created (see Fig. 3a) and panoramic images (see Fig. 4b for an example) were rendered as described in Dittmar et al. (2010). Similarities between views were computed using the minimum image distance over all orientations (see Fig. 4). The image distance function we used was the sum of the squared pixel differences, which was calculated after conversion of the rendered colour images to grey images (and thus yellow and blue stripes were converted to grey stripes of different brightness); orientations were tested in steps of $1^{\circ}$. We also included a weighting factor that compensated for the distortions of the viewing sphere introduced by the equirectangular mapping of the panoramic images. Reference snapshots were rendered at the decision lines $(8 \mathrm{~cm}$ in front of the food hole) oriented towards the food holes (see Fig. $4 \mathrm{~b}$ as an example). These oriented snapshots were compared to images rendered at positions on a two-dimensional grid of $2 \mathrm{~cm}$ spacing, $10 \mathrm{~cm}$ above floor level.

\section{RESULTS AND DISCUSSION}

\section{Experiment 1 'Local Cues Only'}

In this experiment bees could only use the local cues provided by the box, since the lid prevented the bees from using external visual cues. Bumblebees and honeybees both distinguished the two diagonally opposite corners ( 1 and 3 ) from the neighbouring corners ( 2 and 4 ) as the corner choices were not equally distributed in all conditions (Figs $1 \mathrm{a}-\mathrm{c}, 2 \mathrm{a}-\mathrm{c}$; Pearson chi-square test: $P<0.001$ ). However, they could not distinguish between the rewarded corner and the diagonally opposite one (during training and during a control test with the box rotated by $180^{\circ}$; Pearson chi-square test: $P>0.05)$.

\section{Bees confuse the diagonally opposite corners}

From within the box these two corners looked very similar because of the geometry of space (i.e. angular and metric relations between the corners and sense of left-right relations) and the local colour cues (to the left of the food hole a yellow stripe and to the right a blue stripe, see for example Figs $1 \mathrm{a}, 2 \mathrm{a}$ ). Thus, as shown for many other animals in similar experimental environments (e.g. Cheng, 1986; Kelly \& Spetch, 2001; Sovrano, Bisazza, \& Vallortigara, 2002; Wystrach \& Beugnon, 2009), including recently for bumblebees in a different and five times smaller box (Sovrano et al., 2012, 2013), bees also made rotational errors in our rectangular box. Note that bees did not always land in the corner where they first crossed the decision line. Hence, the percentage of first landings exceeded the percentage of first choices for some corners. The first landings of honeybees during training were, in contrast to the first corner choices, not symmetrically distributed between the two diagonally opposite corners (Fig. 2a). The honeybees might have used near-range cues possibly associated with the sugar-water provided in the rewarded food hole (e.g. they might have probed the food holes with their antennae before deciding whether they should land). This effect is not visible in tests in which no sugarwater was provided (Fig. 2b). Even though similar experiments have shown that bees can use magnetic cues under some experimental conditions (Collett \& Baron, 1994; Frier, Edwards, Smith, Neale, \& Collett, 1996), we found no evidence that the bees used magnetic compass information in our experiments, since this would have allowed them to distinguish corner 1 from corner 3 .

\section{Bees rely predominantly on pictorial cues}

To test whether the bees predominantly used geometric information to distinguish between the neighbouring corners, we exchanged the coloured stripes adjacent to the food hole (see Figs $1 c, 2 c$ ). If the bees mostly relied on geometric information, they would still search in corner 1 and the diagonally opposite corner 3 since these corners have a long wall on the left and a short wall on the right like the rewarded corner during training. We found instead that the bees changed their choice behaviour and now equally preferred corners 2 and 4 (Pearson chi-square test: $P>0.05$ ), which provided the same colour configuration as the rewarded corner (Figs 1c, 2c). The behaviour of honeybees and 
bumblebees under our experimental conditions was very similar. Thus, we conclude that bumblebees and honeybees relied more strongly on the colour configuration than on geometric information under our experimental conditions. In agreement with our findings, Sovrano et al. (2013) recently reported that bumblebees predominantly relied on featural cues when there was a conflict between geometrical and featural cues, which the authors created by swapping four cardboard panels with different colours and patterns in a small $(200 \mathrm{~mm} \times 96 \mathrm{~mm})$ rectangular box. Sovrano et al. (2013) also suggested that their observations might be explained by matching of panoramic images, a simple view-based model that, as we show below, is capable of describing the corner choices of bees in all our experiments presented so far.

In recent years, model simulations and behavioural experiments in ants have shown that rotational errors in rectangular arenas can be parsimoniously explained by view-based strategies without explicit representation of geometry (Stürzl et al., 2008; Wystrach et al., 2011; Wystrach \& Beugnon, 2009). The choice behaviour of the bees in our experimental set-up agrees very well with a simple image-matching model (see Fig. 4). Based on the assumption that the bees memorize a panoramic image at the goal location (at a distance of $8 \mathrm{~cm}$ in front of the food hole) oriented towards the rewarded food hole and search at locations where the similarity to the memorized image is high, we calculated the similarity between the memorized image and the local views for different locations in the box (Fig. 4). The similarity map in Fig. 4a shows that the similarity is high (or, equivalently, the weighted sum of the squared pixel differences is low) at the diagonally opposite corners 1 and 3 . In the similarity map corresponding to test 2 (test with colour stripes interchanged) the positions of high similarity have moved to corners 2 and 4, while less pronounced local minima can be found at the geometrically correct corners 1 and 3 (see Fig. 4b). This is because the matching of the vertical stripes close to the food hole dominates image similarity in our experimental flight arena. Thus, image matching can explain the observation that bees prefer to choose corners that provide the same colour distribution as the rewarded corner during training. Note that the effect of features on image similarity depends on multiple factors, such as contrast and retinal size. Therefore, swapping of coloured features can have a different effect in a different environment (see Stürzl et al., 2008 for further discussion).

How could image similarity guide the bees towards the positions with the best matching views? It has been shown that image similarity usually changes smoothly in the neighbourhood of local extrema and, thus, the change in image similarity or its gradient could be used to determine the heading direction (gradient descent model, Zeil, Hofmann, \& Chahl, 2003). It is also obvious from Fig. 3 that the orientations of the best matching views (depicted by arrows) often point in the correct directions and could be exploited by the insects to determine the direction to the goal (visual compass model, Graham, Philippides, \& Baddeley, 2010; Wystrach et al., 2011).

Actually, a combination of both methods would be advantageous because they are complementary with respect to the reliability of the heading direction they provide. For instance, the orientation estimated by the visual compass model is very reliable for areas between the centre of the box and the corners where the image similarity surface is very flat and has spurious local minima resulting in erroneous heading information. By contrast, the visual compass model is not useful for guidance in the central part of the box where arrows basically point along a vortex away from the corners and also close to the walls of the box. Here image similarity is very low and gradient information correctly points away from the centre and the walls. Hence, a simple and very efficient method for locating the corners would be to move according to the best matching viewing direction as long as the direction is not contradicted by a strong decrease in image similarity and as long as image similarity increases in the long run. A strong decrease in image similarity would indicate that the heading direction should be changed according to the image similarity gradient.

Such a simple view-based model would have to be modified to account for the fact that bees do not get stuck in the local optima at the corners of the box, for example by performing U-turns directed towards another corner of the box in a similar way as described by Wystrach and Beugnon (2009) for ants.

\section{Experiment 2 'Additional Extrabox Cues’}

The bees should be able to distinguish the two corners of similar appearance if they have extra information about their global orientation. In the second experiment we therefore investigated whether and how bees use extrabox cues to identify the rewarded corner. We tested the bees in the same rectangular box but this time without the lid and with visual cues outside the box (see Fig. 3 and Methods for details).

\section{Bees can use extra-arena cues}

With extrabox cues, the bees preferred only the rewarded corner (about $90 \%$ of the choices; see Figs 1d,e, 2d,e) and displayed almost no rotational errors (Pearson chi-square test: $P<0.05$ ).

\section{Extrabox cues override local pictorial cues}

As an additional test, we rotated the extrabox cues by $180^{\circ}$ with respect to the box (Fig. 1f, performed only with bumblebees as they were tested only with two external visual cues in a windowless room). The bumblebees then preferred the correct corner with respect to the external visual cues, which was rotated by $180^{\circ}$ with respect to the room (Pearson chi-square test: $P<0.05$ ). Even when we exchanged the local colour cues, the bees still preferred the correct corner with respect to the external world (Pearson chisquare test: $P<0.05$, see Fig. 2 f; performed only with honeybees). This shows that the bees relied more strongly on the extrabox cues than on the local cues provided in the box, as they ignored the wrong colour cues at the preferred corner. These findings are in agreement with an earlier experiment (Collett \& Kelber, 1988) in which bees were trained outdoors to collect food from two platforms $40 \mathrm{~m}$ apart, which were defined by local landmarks of different colours and shapes (yellow cylinders versus blue triangles). When these landmarks were exchanged between platforms, bees searched on each platform as defined by the more distant surroundings, that is, as if the landmarks had not been swapped. Bees approached the goal from within their familiar natural environment in this experiment. Despite the swapping of local landmarks, image similarities might thus still have been highest at the locations where the bees actually searched, owing to numerous dominating features in the surrounding natural scene. This was different in our experiment, in which bees started from inside the box, where the panorama did not provide large unambiguous cues.

Indeed, we cannot explain how bees are able to identify the correct corner without making rotational errors by matching panoramic views as seen from any position inside the box. The similarity map for the bumblebee box and the two extrabox cues shows that the similarity was still highest at the rewarded and the diagonally opposite corner (see Fig. 4c). This is because the cues covered only a small part of the visual field (see 'bee-eye views', Fig. 3c). It has been reported previously that ants displayed rotational errors when features on the box wall covered only about $7 \%$; however, when the feature wall covered $35 \%$ of the azimuth (resulting in strong mismatches between the different corners), the 
resulting mismatches were large enough for the ants to avoid errors (Wystrach et al., 2011).

Since bees do not display rotational errors when room cues are available, they must have used a different strategy to locate the correct corner. When starting from the centre of the box, the cues were well visible in their upper field of view and they could have used them as compass cues to determine their flight direction and then locate the corner by increasing the similarity to their memorized view. Seen from the centre of the box the larger cue covered about $20^{\circ}$ in elevation and $30^{\circ}$ in azimuth. This should be large enough to be detectable as the visual resolution of the bee's compound eye has been behaviourally estimated to be in the range of 2-5 (Giurfa, Vorobyev, Kevan, \& Menzel, 1996; Macuda, Gegear, Laverty, \& Timney, 2001; Spaethe \& Chittka, 2003; Srinivasan \& Lehrer, 1988; Wertlen, Niggebrügge, Vorobyev, \& Hempel de Ibarra, 2008). The black ceiling, used in the honeybee experiments, provided an even larger directional cue. It covered about $55^{\circ}$ in the horizontal and $45^{\circ}$ in the vertical direction as seen from the centre of the box and was also visible from a position in the rewarded corner (see Fig. 3c). Although the cues were large enough to be detectable, the bees did not seem to be able to use them directly for locating the rewarded corner. We observed that the bees chose a different strategy to identify the correct corner. Most honeybees and all bumblebees left the box before flying to the correct food hole. This resulted in a much longer decision time than in the experiments without room cues. We measured the flight duration of bumblebees as time between departure and the first landing. Bumblebee flights without local cues $(N=59)$ had a median duration of $9 \mathrm{~s}$ (IQR (interquartile range) $=16 \mathrm{~s}$ ) and those bumblebees that had extrabox cues available $((n=60)$ : median $=23 \mathrm{~s}, \mathrm{IQR}=43.5 \mathrm{~s}$ ) took significantly longer (Wilcoxon rank sum test: $P<0.001$ ). When the bees left the box, we observed they flew towards the visual cue outside the box located in the direction of the rewarded corner and re-entered it at the side of the correct corner. The distant visual cues seemed to determine where they first entered the box. During training, $77.8 \%(N=7, n=66)$ of the bumblebees entered the box at the side where the visual cues were presented, $66.6 \%(N=3, n=7)$ during the control test (see Fig. 1e) and $71.4 \%(N=5, n=11)$ during the test with the visual cues rotated by $180^{\circ}$ (see Fig. 1f).

This strategy seemed to be necessary to identify the correct corner in the box: the few honeybees that did not leave the box (17.5\% of the flights; not included in the figures) continued to make rotational errors $(N=3, n=14$ corner choices: rewarded corner $53 \%$; diagonally opposite corner $47 \%$, other corners $0 \%$, as predicted by our simulations.

\section{GENERAL DISCUSSION}

How animals deal with ambiguous information often provides insights into how their brains process information and plan motor actions. In this study we investigated how bees distinguish between two similar-looking places. We found that bees confused the two diagonally opposite corners of a rectangular box that provided symmetrically arranged colour cues. When we then swapped cues between corners, bees preferred corners that had cues similar to the trained corner, even when the geometric relations were incorrect. Apparently, they relied on views rather than on a separate geometric representation, which we corroborated by computer simulations.

Up to this point, all our findings can be explained by a viewbased matching strategy, thus confirming results from previous studies (e.g. Sovrano et al., 2012, 2013; Wystrach \& Beugnon, 2009). This does not rule out, of course, that bees use additional spatial representations as well. It has been shown that bees can use depth information estimated from optical flow, that is, from shifts in the retinal images caused by movement of the observer, for localizing a place (Dittmar et al., 2010). Young children and chicks, Gallus gallus domesticus, seem to use depth information or other nonpictorial representations to reorient in environments with rectangular arrangements that provide subtle three-dimensional cues (Lee \& Spelke, 2011; Lee, Spelke, \& Vallortigara, 2012). Note that view-based matching, that is, matching of retinotopic information, is not limited to matching of images but can also be applied to other views that contain, for instance, edges (Stürzl et al., 2008) or depth information (Dittmar et al., 2010). Since all results of the experiments with 'in-box cues only' can be described by image matching we did not test more complicated view-based matching models.

We extended the simple and well-known 'animal in a rectangular box' paradigm by adding distant visual cues outside the box and allowing the animals to approach these cues. Using these cues, bees could in principle resolve the ambiguity between the diagonal, equal-looking corners, even without leaving the box. We found that bees were able to locate the correct corner, and thus distinguish between two places of similar appearance, when visual cues outside the box were provided. In a cue-conflicting task, bees relied even more strongly on these distant cues than on the local colour cues in the box to identify the correct corner. There is evidence that ants can directly use distant panoramic cues that are large enough and not limited to the upper field of view to determine their heading direction (Graham \& Cheng, 2009a, 2009b). Distant visual cues in our study covered only a small area in the upper field of view and image similarity was therefore ambiguous, despite these cues. This was corroborated by computer simulations, which showed that this performance cannot be explained by matching of views from inside the box. How do the bees then solve the task? We allowed bees to explore the different types of cues by letting them fly out of the box and back again. This allowed us to observe and analyse whether and how bees use cues for homing without forcing them to choose one specific navigation strategy.

Indeed, we found that the bees established a different behavioural strategy allowing them to distinguish between the equallooking corners, when distant cues were available. They actively acquired information by leaving the experimental box and flying towards the distant cues. From there they re-entered the arena from the side of the correct corner. Thus, they seemed to integrate the cues outside the box in their route memory. Using such route memories, bees were then able to resolve the ambiguity and distinguish between two similar-looking places. It is known that honeybees can use salient objects as route landmarks to segment the foraging trip by associating a local vector with each landmark (Chittka, Geiger, \& Kunze, 1995; Chittka, Kunze, Shipman, \& Buchmann, 1995; Collett, Baron, \& Sellen, 1996; Collett, Fry, \& Wehner, 1993). To disambiguate the corners of the box used here, they might have memorized a local vector providing flight direction from visual cues outside the box to the rewarded corner. Since, in a natural situation, a goal location is characterized not only by its visual appearance but also by the path towards it, relying on route memories in an ambiguous homing task is, in fact, a clever and robust solution.

\section{Acknowledgments}

We thank Martin Egelhaaf for critically reading the manuscript and Julia Tomberg for help with the experiments. We also thank the referees and the editors for their relevant and useful comments. The study was supported by the Deutsche Forschungsgemeinschaft (Cluster of Excellence ‘Cognitive Interaction Technology'). 


\section{References}

Cartwright, B. A., \& Collett, T. S. (1983). Landmark learning in bees - experiments and models. Journal of Comparative Physiology A, 151, 521-543.

Cheng, K. (1986). A purely geometric module in the rat's spatial representation. Cognition, 23, 149-178.

Cheng, K. (2008). Whither geometry? Troubles of the geometric module. Trends in Cognitive Sciences, 12, 355-361.

Cheng, K., \& Gallistel, C. R. (2005). Shape parameters explain data from spatial transformations: comment on Pearce et al. (2004) and Tommasi \& Polli (2004). Journal of Experimental Psychology: Animal Behavior Processes, 31, 254-259.

Cheng, K., \& Newcombe, N. S. (2005). Is there a geometric module for spatial orientation? Squaring theory and evidence. Psychonomic Bulletin E Review, 12 , $1-23$.

Cheung, A., Stürzl, W., Zeil, J., \& Cheng, K. (2008). The information content of panoramic images II: view-based navigation in nonrectangular experimental arenas. Journal of Experimental Psychology: Animal Behavior Processes, 34, 15-30.

Chittka, L., Geiger, K., \& Kunze, J. (1995). The influences of landmarks on distance estimation of honeybees. Animal Behaviour, 50, 23-31.

Chittka, L., Kunze, J., Shipman, C., \& Buchmann, S. L. (1995). The significance of landmarks for path integration of homing honey bee foragers. Naturwissenschaften, 82, 341-343.

Collett, T. S., \& Baron, J. (1994). Biological compasses and the coordinate frame of landmark memories in honeybees. Nature, 368, 137-140.

Collett, T. S., Baron, J., \& Sellen, K. (1996). On the encoding of movement vectors by honeybees. Are distance and direction represented independently? Journal of Comparative Physiology A, 179, 395-406.

Collett, T. S., Fry, S. N., \& Wehner, R. (1993). Sequence learning by honeybees. Journal of Comparative Physiology A, 172, 693-706.

Collett, T. S., \& Kelber, A. (1988). The retrieval of visuo-spatial memories by honeybees. Journal of Comparative Physiology A, 163, 145-150.

Dittmar, L., Stürzl, W., Baird, E., Boeddeker, N., \& Egelhaaf, M. (2010). Goal seeking in honeybees: matching of optic flow snapshots? Journal of Experimental Biology, 213, 2913-2923.

Frier, H., Edwards, E., Smith, C., Neale, S., \& Collett, T. S. (1996). Magnetic compass cues and visual pattern learning in honeybees. Journal of Experimental Biology, 199, 1353-1361.

Giurfa, M., Vorobyev, M., Kevan, P., \& Menzel, R. (1996). Detection of coloured stimuli by honeybees: minimum visual angles and receptor-specific contrasts. Journal of Comparative Physiology A, 178, 699-709.

Graham, P., \& Cheng, K. (2009a). Which portion of the natural panorama is used for view-based navigation in the Australian desert ant? Journal of Comparative Physiology A, 195, 681-689.

Graham, P., \& Cheng, K. (2009b). Ants use the panoramic skyline as a visual cue during navigation. Current Biology, 19, R935-R937.

Graham, P., Philippides, A., \& Baddeley, B. (2010). Animal cognition: multi-modal interactions in ant learning. Current Biology, 20, R639-R640.

Kelly, D. M., \& Spetch, M. L. (2001). Pigeons encode relative geometry. Journal of Experimental Psychology: Animal Behavior Processes, 27, 417-422.

Lee, S. A., \& Spelke, E. S. (2011). Young children reorient by computing layout geometry, not by matching images of the environment. Psychonomic Bulletin $\mathcal{E}$ Review, 18(1), 192-198. http://dx.doi.org/10.3758/s13423-010-0035-z.

Lee, S. A., Spelke, E. S., \& Vallortigara, G. (2012). Chicks, like children, spontaneously reorient by three-dimensional environmental geometry, not by image matching. Biology Letters, 8, 492-494.
Macuda, T., Gegear, R., Laverty, T., \& Timney, B. (2001). Behavioural assessment of visual acuity in bumblebees (Bombus impatiens). Journal of Experimental Biology, 204, 559-564.

Pearce, J. M., Good, M. A., Jones, P. M., \& McGregor, A. (2004). Transfer of spatial behavior between different environments: implications for theories of spatial learning and for the role of the hippocampus in spatial learning. Journal of Experimental Psychology: Animal Behavior Processes, 30, 135-147.

Pecchia, T., \& Vallortigara, G. (2010). View-based strategy for spatial reorientation by geometry. Journal of Experimental Biology, 213, 2987-2996.

Pecchia, T., \& Vallortigara, G. (2012). Spatial reorientation by geometry with freestanding objects and extended surfaces: a unifying view. Proceedings of the Royal Society B, 279, 2228-2236.

Sheynikhovich, D., Chavarriaga, R., Strösslin, T., Arleo, A., \& Gerstner, W. (2009). Is there a geometric module for spatial orientation? Insights from a rodent navigation model. Psychological Review, 116, 540-566.

Sovrano, V. A., Bisazza, A., \& Vallortigara, G. (2002). Modularity and spatial reorientation in a simple mind: encoding of geometric and nongeometric properties of a spatial environment by fish. Cognition, 85, B51-B59.

Sovrano, V. A., Rigosi, E., \& Vallortigara, G. (2012). Spatial reorientation by geometry in bumblebees. PLoS One, 7, e37449.

Sovrano, V. A., Potrich, D., \& Vallortigara, G. (2013). Learning of geometry and features in bumblebees (Bombus terrestris). Journal of Comparative Psychology, $127,312-318$

Spaethe, J., \& Chittka, L. (2003). Interindividual variation of eye optics and single object resolution in bumblebees. Journal of Experimental Biology, 206, 3447-3453.

Srinivasan, M. V., \& Lehrer, M. (1988). Spatial acuity of honeybee vision and its spectral properties. Journal of Comparative Physiology A, 162, 159-172.

Stürzl, W., Boeddeker, N., Dittmar, L., \& Egelhaaf, M. (2010). Mimicking honeybee eyes with a $280^{\circ}$ field of view catadioptric imaging system. Bioinspiration $\mathcal{E}$ Biomimetics, 5, 036002.

Stürzl, W., Cheung, A., Zeil, J., \& Cheng, K. (2008). The information content of panoramic images I: the rotational errors and the similarity of views in a rectangular experimental arenas. Journal of Experimental Psychology: Animal Behavior Processes, 34, 1-14.

Tommasi, L., Chiandetti, C., Pecchia, T., Sovrano, V. A., \& Vallortigara, G. (2012). From natural geometry to spatial cognition. Neuroscience E'Biobehavioral Reviews, 36, 799-824.

Tommasi, L., \& Polli, C. (2004). Representation of two geometric features of the environment in the domestic chick (Gallus gallus). Animal Cognition, 7, 53-59.

Vallortigara, G. (2009). Animals as natural geometers. In L. Tommasi, M. A. Peterson, \& L. Nadel (Eds.), Cognitive biology, evolutionary and developmental perspectives on mind, brain and behavior (pp. 83-104). Cambridge, MA, U.S.A.: MIT Press.

Wang, R. F., \& Spelke, E. S. (2002). Human spatial representation: insights from animals. Trends in Cognitive Sciences, 6, 376-382.

Wertlen, A. M., Niggebrügge, C., Vorobyev, M., \& Hempel de Ibarra, N. (2008). Detection of patches of coloured discs by bees. Journal of Experimental Biology, 211, 2101-2104.

Wystrach, A., \& Beugnon, G. (2009). Ants learn geometry and feature. Current Biology, 19, 1-9.

Wystrach, A., Cheng, K., Sosa, S., \& Beugnon, G. (2011). Geometry, features, and panoramic views: ants in rectangular arenas. Journal of Experimental Psychology: Animal Behavior Processes, 37, 420-435.

Zeil, J. (2012). Visual homing: an insect perspective. Current Opinion in Neurobiology, 22, $1-9$.

Zeil, J., Hofmann, M. I., \& Chahl, J. S. (2003). Catchment areas of panoramic snapshots in outdoor scenes. Journal of the Optical Society of America A, 20, 450-469. 